\title{
Original
}

\section{Bone Regeneration by Demineralized Dentin Matrix in Skull Defects of Rats}

\author{
Ken Togari ${ }^{1)}$, Ken Miyazawa ${ }^{1)}$, Keisuke Yagihashi ${ }^{1)}$, Masako Tabuchi ${ }^{1)}$ \\ Hatsuhiko Maeda'), Tatsushi Kawai ${ }^{3)}$ and Shigemi Goto ${ }^{1)}$
}

\author{
${ }^{1)}$ Department of Orthodontics, School of Dentistry, Aichi Gakuin University, Nagoya, Japan \\ ${ }^{2)}$ Department of Oral Pathology, School of Dentistry, Aichi Gakuin University, Nagoya, Japan \\ ${ }^{3}$ Department of Dental Materials Science, School of Dentistry, Aichi Gakuin University, Nagoya, Japan \\ (Accepted for publication, Nobember 1, 2011)
}

\begin{abstract}
Alveolar bone defects may result in functional and morphological abnormalities. In such cases, bone can be regenerated by grafting fresh autogenous bone into the defect. Subsequently, we focused on the dentin of third molars, which are usually discarded after extraction, or teeth removed faor orthodontic therapy. As in bone, type I collagen is the main component of the organic matrix in dentin. Also, it has been proven that the organic matrix of dentin contains osteoinductive bone morphogenetic protein (BMP). It would be safe to perform grafting with the dentin of extracted teeth used as an autogenous bone substitute. Therefore, the objective of the present study was to reuse extracted teeth as a bone substitute material. Demineralized dentin matrix (DDM), an organic material derived from the dentine of bovine teeth, was grafted in rat skull defects to determine its bone regeneration ability and the possibility of its use as a bone substitute. In the present study, new bone formed from the defect margin in the control group, but in the graft group, new bone formation occurred from individual DDM granules within the defect, and not just from the margin. At Week 8, defect repair was limited in the control group. In contrast, most of the defect was covered by osseous tissue in the graft group. In conclusion, DDM may be a useful bone substitute that serves as a scaffold for bone regeneration by inducing a high level of new bone formation soon after surgery.
\end{abstract}

Key words: Bone regeneration, Demineralized dentin matrix, BMPs, Bone substitute, Micro CT

\section{Introduction}

Alveolar bone defects may result in functional and morphological abnormalities. In such cases, bone can be regenerated by grafting fresh autogenous bone into the bone defect. Fresh autogenous bone grafting is widely performed because it has no risk of infection or rejection, and favorable results have been obtained ${ }^{1)}$. However, harvesting autogenous bone has disadvantages such as surgical invasiveness, wound infection, chronic pain, nerve paralysis and scarring ${ }^{2}$. Also, the volume of bone that can be harvested is limited. Therefore, methods that use a bone substitute material instead of autogenous bone are being investigated.

At present, bone substitute materials consisting of natural bone tissue, such as allogeneic and xenogenic bone, are used for bone grafting. Hydroxyapatite, an artificial biomaterial, is also widely used because of its high tissue affinity and osteoconductive properties ${ }^{3)}$. However, hydroxyapatite is nonresorbable and

Correspondence to: Dr. Ken Miyazawa, DDS, PhD, Department of Orthodontics, School of Dentistry, Aichi Gakuin University, 2-11 SuemoriDori, Chikusa-ku, Nagoya 464-8651, Japan, Phone: +81-52-751-7181, Ext. 5378, Fax: +81-52-751-8900, E-mail: miyaken@dpc.aichigakuin.ac.jp remains in the body for a long period of time ${ }^{4)}$. Hydroxyapatite acts as a scaffold for bone formation, but does not induce new bone tissue. Therefore, bone formation may be delayed ${ }^{5)}$.

Subsequently, we focused on the dentin of third molars that are usually discarded after extraction or teeth removed for orthodontic therapy. As in bone, type I collagen is the main component-accounting for about $20 \%$ by weight — of the organic matrix in dentin. Also, it has been proven that the organic matrix in dentin contains osteoinductive bone morphogenetic protein $(\mathrm{BMP})^{6}$. We have demonstrated that demineralized dentin matrix (DDM) acts as a scaffold for repair of articular cartilage defects ${ }^{7}$. Moreover, recent studies examine a multi-component technique consisting of a novel side population of multi-purpose stem cells cultured on demineralized bone and dentin matrix, for tissue engineering applications ${ }^{8-13)}$. The dentin of extracted teeth used as an autogenous bone substitute would be safe for grafting. Therefore, the objective of the present study was to reuse extracted teeth as a bone substitute material.

DDM, an organic material derived from the dentine of bovine teeth, was grafted in rat skull defects to determine its bone regeneration ability and the possibility of its use as a bone 
A

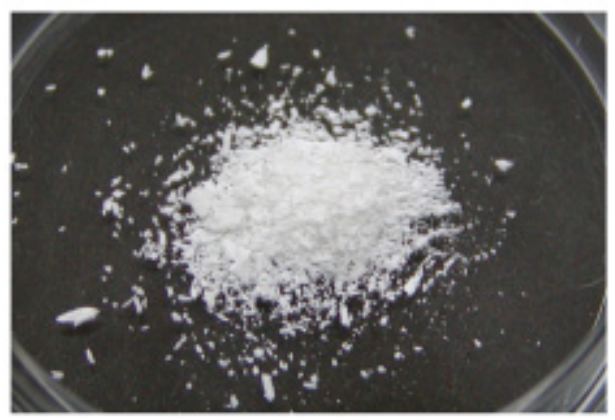

B



Figure 1A. DDM powder following lyophilization

substitute.

\section{Materials and Methods}

\section{Animals}

One hundred and twenty 5-week-old male Wistar rats (CLEA Japan, Inc., Tokyo, Japan) weighing about 100 g were divided into two groups: control group $(n=60)$ and graft group $(n=60)$. Each group was subdivided into the following five groups based on the time after surgery: Weeks 1, 2, 4, 6 and 8 ( $\mathrm{n}=12$ each). The rats were maintained under the same conditions, and they had free access to food and water. The present study was conducted according to the Animal Experiment guidelines of Aichi Gakuin University School of Dentistry.

\section{DDM preparation}

The DDM used in the study was prepared from bovine lower anterior teeth by slightly modifying the method of Urist et al. ${ }^{14)}$. Bovine lower anterior teeth were extracted, and after removing the surrounding soft tissue and dental pulp, they were frozen at $80^{\circ} \mathrm{C}$ for $24 \mathrm{~h}$. After complete demineralization $(0.6 \mathrm{~N} \mathrm{HCl}, 1$ week), the teeth were washed using distilled water and defatted for $24 \mathrm{~h}$ (chloroform/methanol). The teeth were then lyophilized, and DDM was pulverized using FREEZE/MILL (SPEC Industries Inc., MA, USA). In order to make the particle size even, DDM with particle sizes of 250 - 500 mm was collected by sieving (Fig. 1) ${ }^{7)}$.

\section{Surgical methods and procedures}

The rats were made to inhale diethyl ether, and under general anesthesia, the head was shaved and the surgical field was sterilized. An incision was made on the head to expose the parietal bone covered by the periosteum. Using a bur and micromotor, a 4.3-mm round bone defect was prepared under continuous irrigation with sterile physiological saline. In the DDM group, 10 mg of DDM was placed in the defect, and in the control group, no material was placed in the defect. The periosteum was replaced, and the scalp was sutured using nylon and closed with surgical adhesive.

For labeling the calcification rate, $0.1 \mathrm{ml} / 100 \mathrm{~g}$ of calcein was administered intraperitoneally 5 and 1 days before dissection. Calcein was dissolved in $2 \%$ sodium hydrogen carbonate solution to a concentration of $8 \mathrm{mg} / \mathrm{kg}$ (b.w.) $/ \mathrm{ml}$. The rats were sacrificed $1,2,4,6$ or 8 weeks after surgery and the skull was dissected.

\section{Bone volume (BV) and Bone surface (BS) measurement}

Each rat skull was analyzed using a micro CT machine (SMX225CT, SHIMADZU CORPORATION, Kyoto, Japan). Using image analysis software (TRI/3D-BON, Ratoc System Engineering Co., Ltd., Tokyo, Japan), stereoscopic images were constructed and morphological analysis was conducted. Based on CT values, soft tissue, DDM and hard tissue were differentiated, and only hard tissue was subjected to morphological analysis to measure bone volume (BV) and bone surface (BS) ${ }^{15)}$.

\section{Histological observation}

Specimens for histological observation were prepared as follows. Tissue samples were demineralized using 10\% EDTA and embedded in paraffin according to the conventional method. Then, 5-ìm-thick serial sections were prepared, stained using hematoxylin-eosin, and observed under a light microscope (ECLIPSE E600, NIKON CORPORATION, Tokyo, Japan).

\section{Calcification rate measurement}

In order to measure calcification rate, GMA-embedded polished samples were prepared. Then the samples were observed under an epifluorescence microscope (ECLIPSE E600, Nikon), and calcein double lines labeled in the newly formed bone within the defect area were photographed under $\times 4$ magnification. Using the calcein-labeled samples, calcification rate was measured according to the conventional method ${ }^{17,18)}$.

\section{Statistical analysis}

Numerical data were expressed as mean \pm standard deviation, and Student t-test was used for statistical analysis. 
Ken Togari et al.: Bone Regeneration by DDM in Skull Defects of Rats

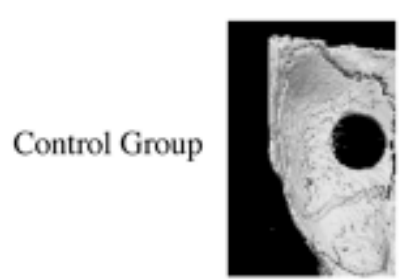

Week 1

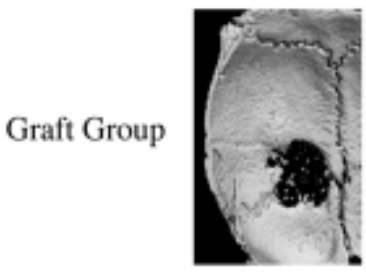

A



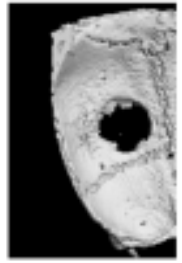

Week 2

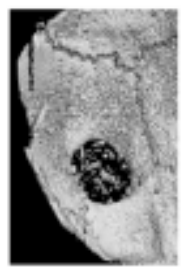

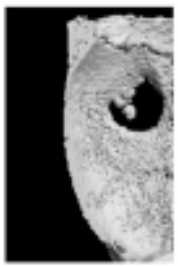

Week 6

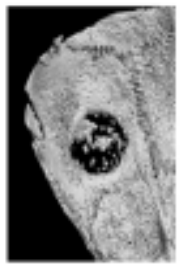

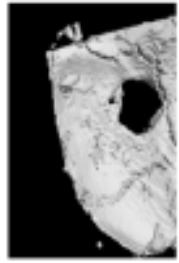

Week 8

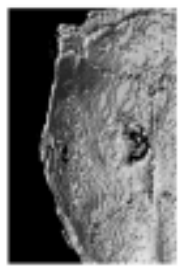

Figure 2. Stereoscopic micro CT images of bone defects.

B

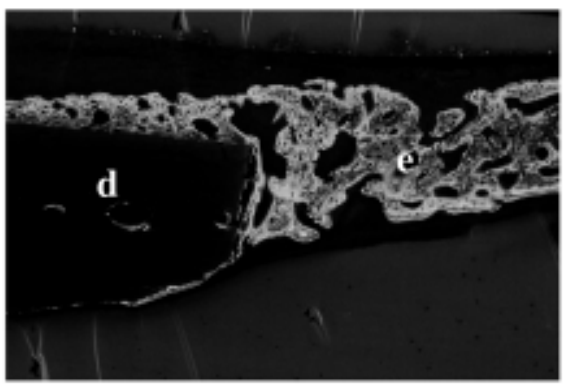

Figure 3A. Fluorescent microscopy image at Week 4 for the control group $(4 \times 10)$. In the skull margin of the bone defect (d), slight calcein labeling is seen (e).

Figure 3B. Fluorescent microscopy image at Week 4 for the DDM graft group $(4 \times 10)$. From the skull margin of the bone defect (d) to the center, active calcein labeling is seen (e).

\section{Results}

\section{Stereoscopic image analysis by micro CT}

Figure 2 shows the chronological changes in bone defects as ascertained by micro CT stereoscopic images. In the control group at Week 1 and 2, slight formation of new bone-like tissue was seen from the margin of the bone defect. When compared to Weeks 1 and 2, the amount of new bone-like tissue in the margin was greater after Week 4. However even at Week 8, defect repair was limited.

On the other hand, in the graft group, new bone-like tissue was actively formed at Weeks 1 and 2, and new bone-like tissue was seen not only at the margin of the defect but also from different locations inside the defect simultaneously. After Week 4, the formation of new bone-like tissue was even more significant at the margin and center, and at Week 8, most of the defect was covered by osseous tissue.

\section{Measurement of calcification rate}

Figure 3 shows the fluorescent microscopic images at Week 4. In the control group, only a small amount of labeling was seen at the defect margin at Week 4, but in the graft group, active labeling was seen from the defect margin to the center at Week 4 .

Figure 4 shows the chronological changes in calcification rate at the defect. The chronological changes in calcification rate for the two groups were mostly comparable. In both groups, calcification rate significantly increased from Weeks 1 to $2(*$ : p $<0.05$ ) and from Weeks 2 to $4(* *: \mathrm{p}<0.01)$. However, from Weeks 4 to 6 , the calcification rate significantly decreased (**: p $<0.01$ ). No significant changes were seen from Weeks 6 to 8 . No significant difference was seen in calcification rate between the two groups at any time period.

\section{BS measurement}

Figure 5 shows the chronological changes in BS at the bone defect. In the control group, BS markedly increased from Weeks 2 to 4 . No significant changes were seen in BS from Weeks 1 to 2 or after Week 4 , but a significant increase was observed from Weeks 2 to 4 (**: p < 0.01).

In the graft group, BS increased with time. No significant 
A
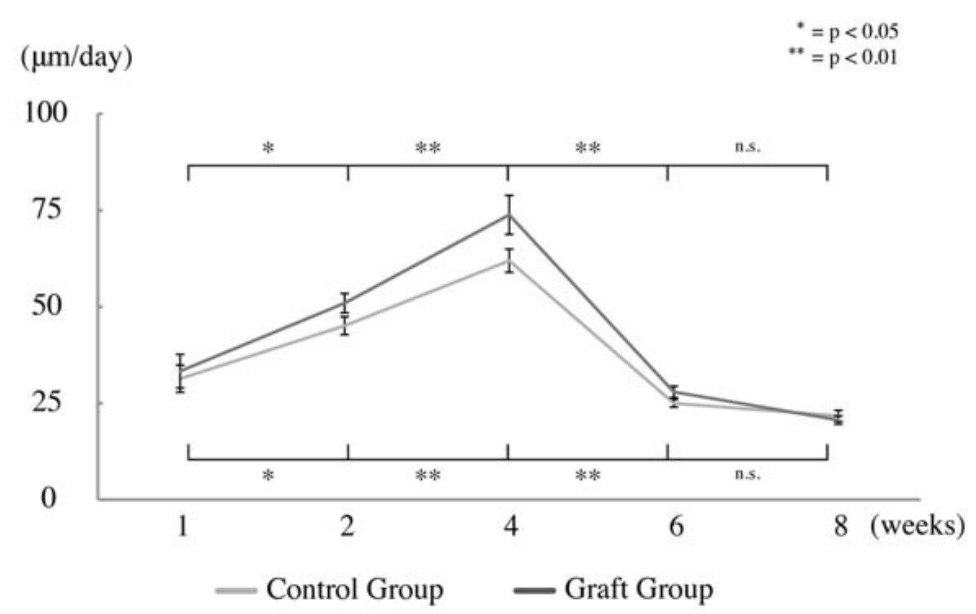

B

$(\mu \mathrm{m} /$ day $)$

\begin{tabular}{|c|c|c|c|c|c|}
\hline $\begin{array}{l}\text { Weeks } \\
\text { after } \\
\text { operation }\end{array}$ & 1 & 2 & 4 & 6 & 8 \\
\hline $\begin{array}{l}\text { Control Group } \\
\text { Graft Group }\end{array}$ & $\left.\begin{array}{l}31.3 \pm 3.5 \\
33.3 \pm 4.4\end{array}\right] \mathrm{ns.}$ & $\left.\begin{array}{l}45.1 \pm 2.3 \\
50.9 \pm 2.5\end{array}\right] \mathrm{ns.}$ & $\left.\begin{array}{l}61.9 \pm 3.0 \\
73.8 \pm 5.1\end{array}\right] \mathrm{ns.}$ & $\left.\begin{array}{l}25.0 \pm 1.0 \\
27.9 \pm 1.5\end{array}\right] \mathrm{ns.}$ & $\left.\begin{array}{l}21.7 \pm 1.4 \\
20.6 \pm 1.1\end{array}\right] \mathrm{ns.}$ \\
\hline
\end{tabular}

Figure 4A. Comparison of changes in calcification rates in each week.

Figure 4B. Comparison of chronological changes in calcification rates of control and graft groups.

A

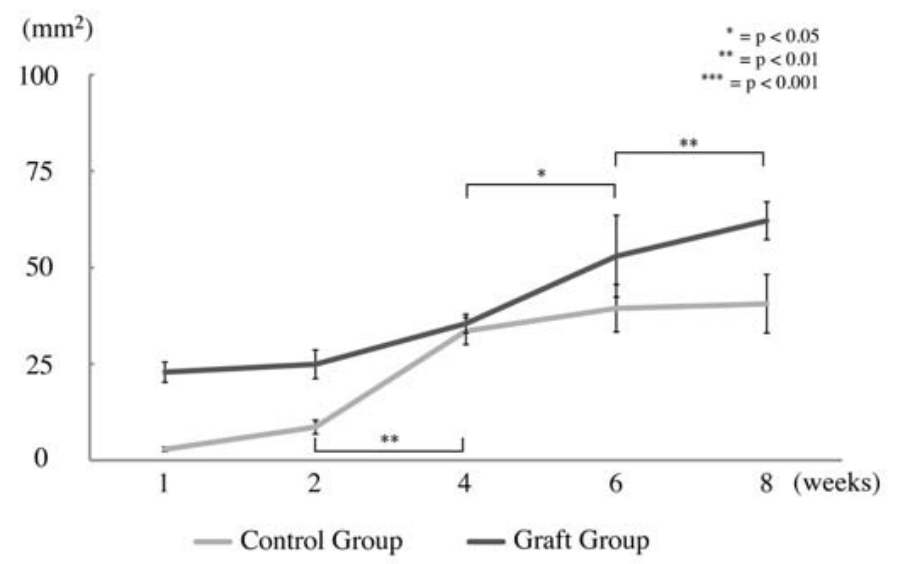

B

$\left(\mathrm{mm}^{2}\right)$

\begin{tabular}{|c|c|c|c|c|c|}
\hline $\begin{array}{l}\text { Weeks } \\
\text { after } \\
\text { operation }\end{array}$ & 1 & 2 & 4 & 6 & 8 \\
\hline Control Group & $2.9 \pm 0.6$ & $8.6 \pm 1.8$ & $33.6 \pm 3.57$ ns. & $39.4 \pm 6.17 \mathrm{~ns}$. & $40.6 \pm 7.67$. \\
\hline Graft Group & $22.9 \pm 2.6$ & $24.9 \pm 3.7]$ & $35.5 \pm 2.4$ & $52.9 \pm 10.6$ & $62.2 \pm 4.9$ \\
\hline
\end{tabular}

Figure 5A. Comparison of changes in BS in each week.

Figure 5B. Comparison of chronological changes in BS of control and graft groups. 
Ken Togari et al.: Bone Regeneration by DDM in Skull Defects of Rats

A

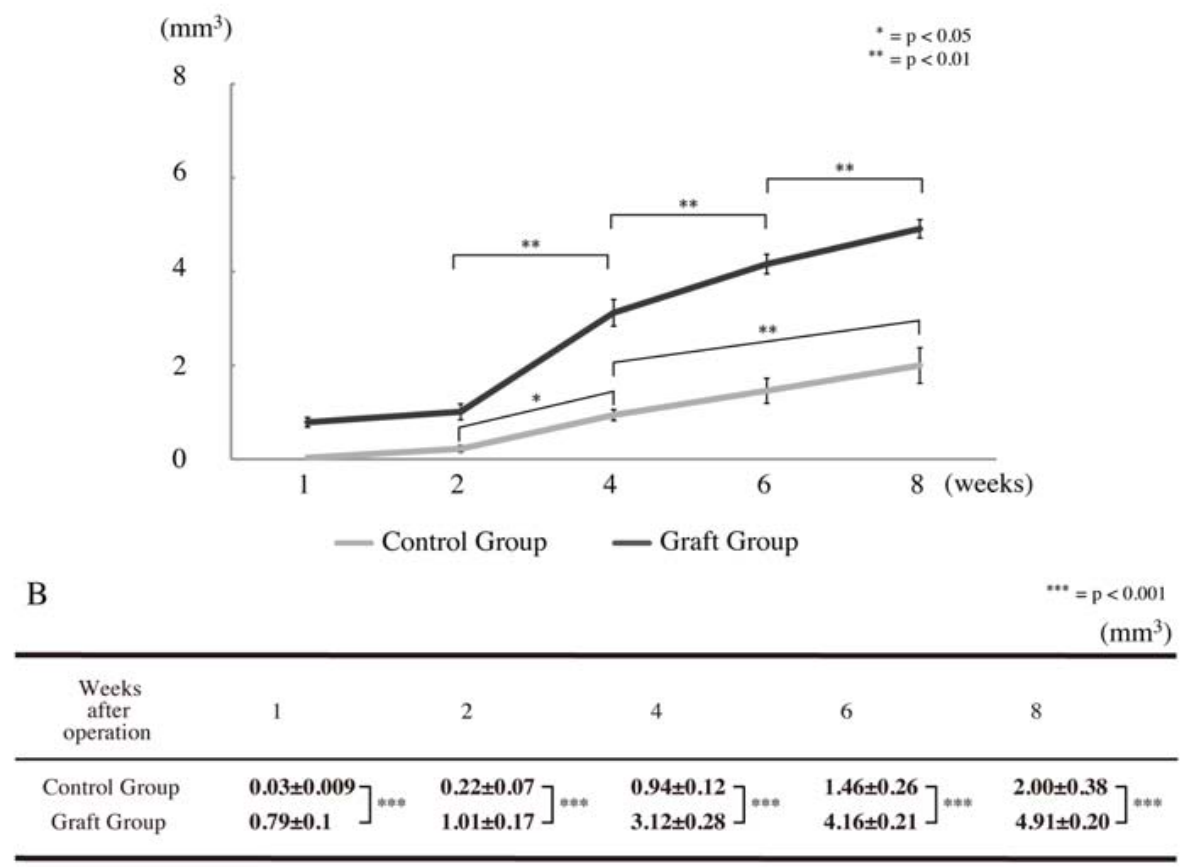

Figure 6A. Comparison of changes in BV in each week.

Figure 6B. Comparison of chronological changes in BV of control and graft groups.

A

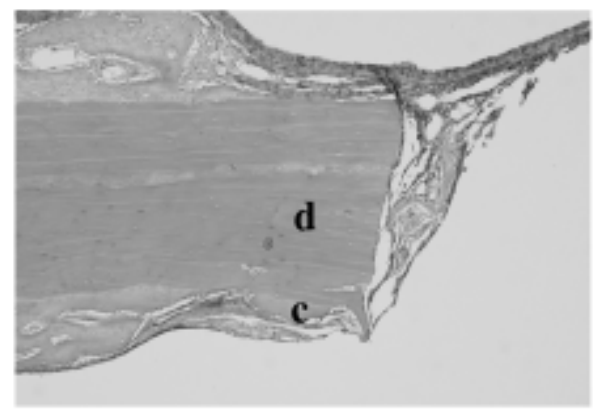

B

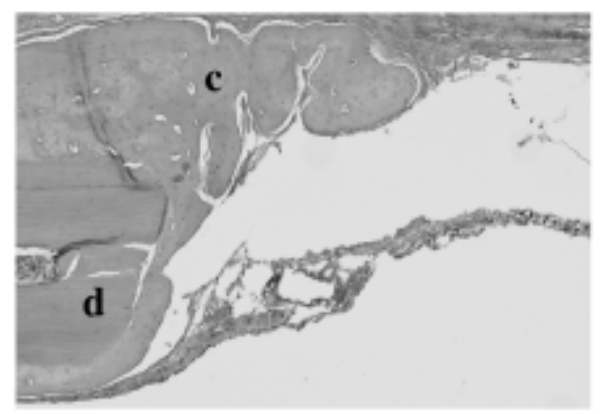

Figure 7A. Histological image at Week 1 for the control group (HE, $4 \times 10)$. In the skull margin of the bone defect (d), small amount of bone formation (c) is observed.

Figure 7B. Histological image at Week 8 for the control group (HE, $4 \times 10$ ). Bone formation (c) is limited to the defect margin (d).

A

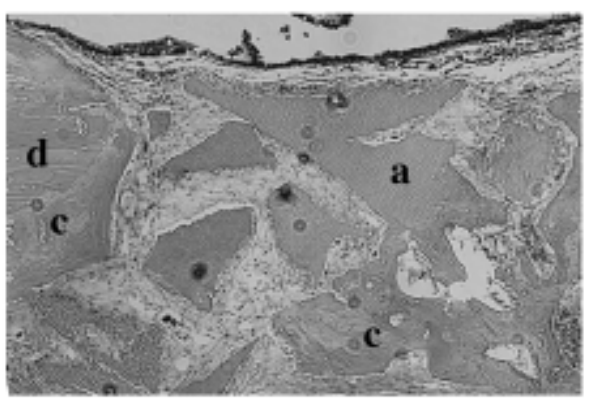

B

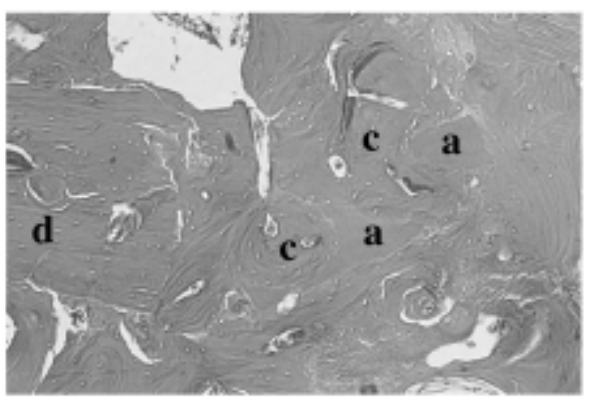

Figure 8A. Histological image at Week 2 for the DDM graft group (HE, $4 \times 10$ ). DDM (a) formed a dense structure with narrow tubes, and formation of bone tissue (c) is seen on the surface of DDM (a).

Figure 8B. Histological image at Week 8 for the DDM graft group (HE, $4 \times 10$ ). Bone tissue (c) is formed at the defect margin (d) and around DDM (a) granules. 
A

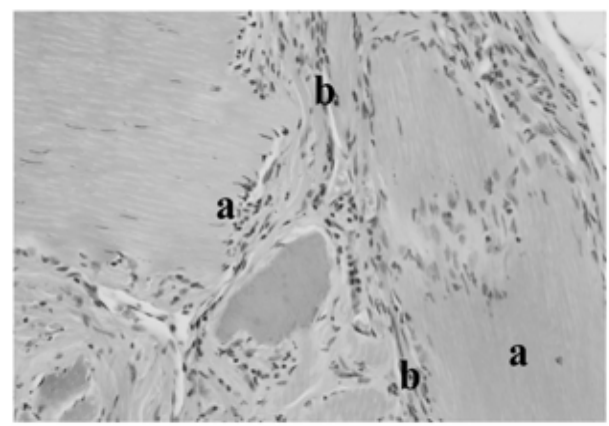

$\mathrm{C}$

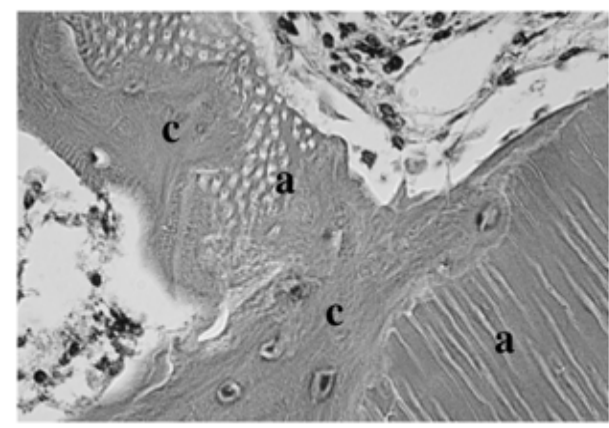

B

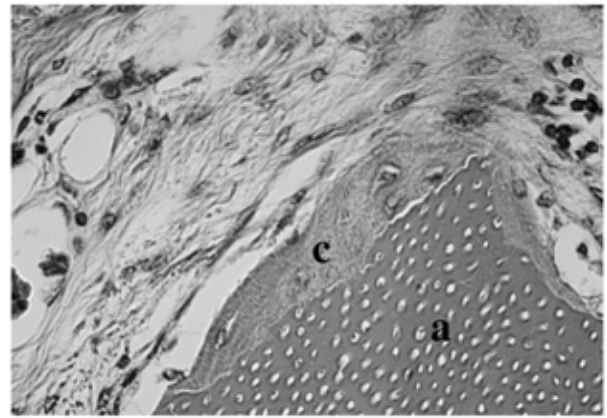

$\mathrm{D}$

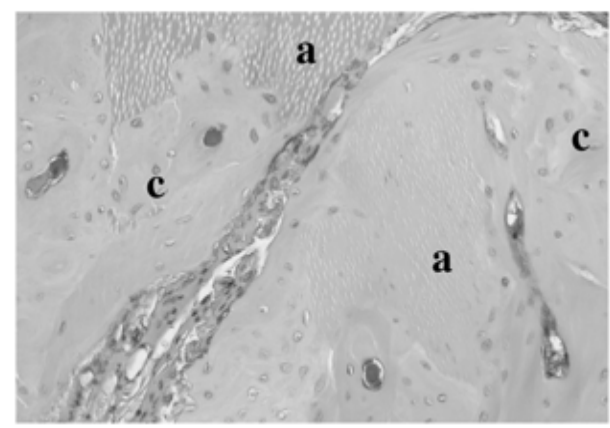

Figure 9A. Histological image at Week 1 for the DDM graft group (HE, 10×10). DDM (a) formed a dense structure with narrow tubes, and formation of osteoid tissue (b) is seen on the surface of DDM (a).

Figure 9B. Histological image at Week 2 for the DDM graft group (HE, 20×10). Formation of bone tissue (c) is seen on the surface of DDM (a).

Figure 9C. Histological image at Week 4 for the DDM graft group (HE, 20×10). Formation of bone tissue (c) bridging DDM (a) is seen.

Figure 9D. Histological image at Week 6 for the DDM graft group (HE, 10×10). Formation of bone tissue (c) surrounding DDM (a) is seen.

changes were seen in BS from Weeks 1 to 4, but a significant increase was seen after Week $4(* *$ : $\mathrm{p}<0.01)$. A significant intergroup difference was seen at Weeks 1 and $2(* *$ : $\mathrm{p}<0.001)$ and Week $8(* *: \mathrm{p}<0.05)$, but not at Weeks 4 and 6. Particularly at Weeks 1 and 2, the BS of the graft group was significantly greater than that of the control group.

\section{$B V$ measurement}

Figure 6 shows the chronological changes in defect BV. In the control group, BV slightly increased with time. No significant change was seen in BV from Weeks 1 to 2 or after Week 4, but a significant increase was seen from Weeks 2 to 4 (*: p < 0.05). However, significant changes were seen from Weeks 4 to 8 (**: p $<0.01)$.

In the graft group, defect BV increased with time. No significant change was seen from Weeks 1 to 2, but significant changes were seen after Week $2(* *: \mathrm{p}<0.01)$. Comparing the two groups, the BV for the graft group was significantly greater at all time points (***: $\mathrm{p}<0.001)$. When compared to the control group, the BV for the graft group was high soon after surgery, and there was about 2.5-fold difference at Week 8.

\section{Histological observation}

Figures 7 and 8 show HE-stained images of both groups. In the control group, a small amount of bone formation was seen at the defect margin from Week 2, but the entire defect was filled with fibrous tissue, and even at Week 8, bone formation was limited to the defect margin (Fig. 7).

In the graft group, the grafted DDM formed a dense structure, and many dentinal tubules were seen. At Week 2, much fibrous tissue was seen among DDM granules, and bone tissue formation was seen not only at the defect margin, but also on the surface of the DDM granules. Also at Week 8, fibrous tissue was mostly absent, and bone tissue had formed at the defect margin and around DDM granules (Fig. 8).

Figure 9 shows enlarged HE images of the graft group. At Week 1, a small amount of osteoid tissue was seen on the surface of DDM (Fig. 9A). At Week 2, bone formation was partially seen on the surface of DDM (Fig. 9B). At Week 4, active bone formation bridging the DDM granules was seen, and the new bone was 
Ken Togari et al.: Bone Regeneration by DDM in Skull Defects of Rats

trabecular in appearance (Fig. 9C). At Weeks 6 and 8, active bone formation was observed, and DDM granules were connected by osseous tissue (Fig. 9D).

\section{Discussion}

\section{Experimental bone defect}

When investigating bone repair in an experimental bone defect, it is necessary to suppress the natural osseous healing of the bone defect. One of the factors affecting bone repair is defect size ${ }^{18)}$, and a critical size defect is an experimental defect where natural osseous healing does not occur ${ }^{19}$. Freeman and Turnbull prepared 2-mm bone defects in the temporal bone of albino Wistar rats weighing $500 \mathrm{~g}$ and reported that the defects were not repaired by new bone even after 12 weeks ${ }^{20,21)}$.

In the present study, a $4.3-\mathrm{mm}$ bone defect involving the periosteum was prepared on the skull of each 5-week-old rat, and even at Week 8, the defect was not repaired, and only minimal new bone formation was seen at the defect margin. When compared to past studies, the defects in the present study were larger, and even after 8 weeks, the size of the defects did not decrease. Therefore, the effects of natural repair by new bone formation were minimal in the present study.

\section{DDM}

The weight ratio of the organic matrix in dentin is about $20 \%$, and as in bone, type I collagen is the main component. The organic matrix includes osteoinductive bone morphogenetic protein (BMP) ${ }^{22)}$. It has also been clarified that demineralized dentin originating from various mammals induces undifferentiated mesenchymal cells to differentiate into osteogenic cells and then into cartilage and bone ${ }^{23}$. Although Reddi documented osteoinduction with particle sizes of 74 to $420 \mu \mathrm{m}^{24,25)}$, our previous study demonstrated particle sizes of 250 to $500 \mu \mathrm{m}$ were highly efficient in osteoinduction ${ }^{7)}$. In the present study, to ensure post-grafting stability, the particle size of DDM was set at 250 to $500 \mu \mathrm{m}$.

\section{Bone generation by DDM grafting}

In the present study, osteoid tissue formation was seen at the bone defect from Week 2 of DDM grafting, and osseous tissue formation was seen at Week 4. At Week 8, the bone defect was filled with osseous tissue. It has been reported that similar in vivo bone formation was seen with demineralized bone and DDM ${ }^{11,26-}$ ${ }^{28)}$. The results of the present study showed that, like demineralized bone, DDM is capable of forming new bone.

With regards to the site of initiation of bone formation, micro CT findings showed that new bone formed from the defect margin in the control group, but in the graft group, new bone formation occurred simultaneously from different locations within the defect, not just from the margin. The BS in the graft group was greater than that in the control group soon after surgery. Also, histological findings showed that bone formation advanced adjacent to DDM. No significant difference was seen in the calcification rate between the graft and control groups. Therefore, when grafting DDM granules in a bone defect, bone formation simultaneously occurs from individual DDM granules, and due to the subsequent increase in bone surface (BS) and bone volume (BV), bone repair advanced faster in the graft group when compared to the control group.

According to unpublished data from our laboratory, the bioactivity of BMP stored at room temperature for more than 10 years has been confirmed. Hence, teeth that are removed at a young age can be processed to prepare DDM, and DDM can be stored frozen using liquid nitrogen for long periods and later thawed to be used as a graft material. Because dentin is autogenous tissue, there is no risk of infection or immunogenic problems, and grafting is possible without invasive harvesting of autogenous bone. Furthermore, because third molars and teeth that are removed for orthodontic therapy are reused, grafting is possible without secondary invasion. Therefore, DDM has advantages such as high bioaffinity, easy harvesting, osteoinduction and no risk of infection, and can be considered as a safe grafting material that may be used instead of autogenous bone graft.

In 1991, a protein with BMP-like activity was isolated from humen dentin, but the $\mathrm{NH}_{2}$-terminal amino acid sequence did not resemble that of the known BMP family ${ }^{29)}$. Moreover, amelogenin-polypeptides with chondrogenic / osteogenic-inducing activity were isolated from normally processed bovine dentine in $1999{ }^{30)}$, but unrelated to therefore, has been unclear until now. We belive that BMP-like molecules and amelogenin-polypeptides might be synergistic factors involved in chondrogenic / osteogenic capability of dentin. Tissue engineering of new bone relies on the combination and application of osteoconductive, osteoinductive, and osteogenic elements. Natural scaffolds, such as demineralized bone and dentin matrix, contain collagenous networks with growth factors such as bone morphogenetic protein-2. Stem cells from readily available sources, including discarded adipose tissue, have the propensity to differentiate into bone. Recent studies examine a multi-component technique consisting of a novel side population of adipose stem cells cultured on demineralized bone and dentin matrix, for tissue engineering applications ${ }^{8-10)}$. In the present study, we investigated the extent to which DDM induces bone repair by implanting DDM derived from bovine teeth grafted in rat skull defects. The new bone formed from the defect margin in the control group, but in the graft group, new bone formation occurred from individual DDM granules within the defect, and not just from the margin. At Week 8, most of the defect was covered by osseous tissue in the graft group. In conclusion, DDM may be a useful bone substitute that serves as a scaffold for bone regeneration by inducing a high level of new bone formation soon after surgery.

\section{Acknowledgments}


J.Hard Tissue Biology Vol. 21(1):25-34, 2012

This study was partially supported by a Grant-in-Aid for Scientific Research from the Ministry of Educations, Science and Culture of Japan (22592305).

\section{References}

1. John RA, Doran ER and Karl AM. The autogenous particulate cancellous bone marrow graft in alveolar clefts. Oral Surg 51: 588-591, 1981

2. Glowacki J, Kaban LB and Murray JE. Application of the biological principle of induced osteogenesis for craniofacial defects. Lancet 1(8227): 959-963, 1981

3. Block MS and Kent JN. Healing of mandibular ridge augmentation using hydroxyapatite with and without autogenous bone in dog. J Oral Maxillofac Surg 43: 3-7, 1985

4. Hoogendoorn HA and Renooij W. Long-term study of large ceramic implants (porous hydroxyapatite) in dog femora. Clin Orthop 187: 281-288, 1984

5. Drobeck HP, Rothstein SS, Gumaer KI, Sherer AD and Slighter RG. Histologic observation of soft tissue responses to implanted, multifaceted particles and discs of hydroxyapatite. J Oral Maxillofac Surg 42: 143-149, 1984

6. Kawai T and Urist MR. Bovine tooth derived bone morphogenetic protein. J Dent Res 68: 1069-1074, 1989

7. Yagihashi K, Miyazawa K, Togari K and Goto S. Demineralized dentin matrix acts as a scaffold for repair of articular cartilage defects. Calcif Tissue Int 84: 210-220, 2009

8. Supronowicz P, Gill E, Trujillo A, Thula T, Zhukauskas R, Ramos $\mathrm{T}$ and Cobb RR. Human adipose-derived side population stem cells cultured on demineralized bone matrix for bone tissue engineering. Tissue Eng Part A 17: 789-798, 2011

9. Murata M. Bone engineering using human demineralized dentin matrix and recombinant human BMP-2. J Hard Tissue Biol 14: 80-81, 2005

10. Rhee SC, Ji YH, Gharibjanian NA, Dhong ES, Park SH and Yoon ES. In vivo evaluation of mixtures of uncultured freshly isolated adipose-derived stem cells and demineralized bone matrix for bone regeneration in a rat critically sized calvarial defect model. Stem Cells Dev 20: 233-242, 2011

11. Gomes MF, Abreu PP, Morosolli AR, Araújo MM and Goulart MG. Densitometric analysis of the autogenous demineralized dentin matrix on the dental socket wound healing process in humans. Braz Oral Res 20: 324-330, 2006

12. Carvalho VA, Tosello Dde O, Salgado MA and Gomes MF. Histomorphometric analysis of homogenous demineralized dentin matrix as osteopromotive material in rabbit mandibles. Int J Oral Maxillofac Implants 19: 679-686, 2004

13. Gomes MF, dos Anjos MJ, Nogueira Tde O and Catanzaro
Guimarães SA. Autogenous demineralized dentin matrix for tissue engineering applications: Radiographic and histomorphometric studies. Int J Oral Maxillofac Implants 17: 488-497, 2002

14. Urist MR and Lietze A. A bovine low molecular weight bone morphogenetic protein (BMP) fraction. Clin Orthop 62: 219232, 1982

15. Kanri Y, Shimazu Y and Aoba T. Use of microfocus X-ray computer tomography for 3D-image construction and quantitative morphoanalysis. J Oral Biosci 46: 67-73, 2004

16. Frost HM. A method of analysis of trabecular bone dynamics. In: Bone Histomorphometry, ed by Meunier PJ, Societe de la nouvelle imprimerie Fournier, Toulouse, 1977, pp445-476

17. Frost HM. Analysis of trabecular bone dynamics. In: Bone histomorphometry: Techniques and Interpretation. ed by R. Recker, CRC Press, Boca Raton, Florida, 1983, pp109-131

18. Hjorting-Hansen E and Andreasen JO. Incomplete bone healing of experimental cavities in dog mandibles. Br J Oral Surg 9: 33-40, 1971

19. Schmitz JP and Hollinge JO. The critical size defect as an experimental model for craniomandibulofacial nonunions. Clin Orthop 205: 299-308, 1986

20. Freeman $\mathrm{E}$ and Turnbull R. The value of osseous coagulum as a graft material. J Periodont Res 8: 229-236, 1973

21. Turnbull RS and Freeman E. Use of wounds in the parietal bone of the rat for evaluating bone marrow for grafting into periodontal defects. J Periodont Res 9: 39-43, 1974

22. Urist MR and Strates B. Bone morphogenetic protein. J Dent Res 50: 1392-1406, 1971

23. Turnbull RS and Freeman E. Use of wounds in the parietal bone of the rat for evaluating bone marrow for grafting into periodontal defects. J Periodont Res 9: 39-43, 1974

24. Reddi AH and Huggins C. Biochemical sequences in the transformation of normal fibroblasts in adolescent rats. Proc Natl Acad Sci USA 69: 1601-1605, 1972

25. Reddi AH and Huggins CB. Influence of geometry of transplant tooth and bone on transformation of fibroblasts. Proc Soc Exp Biol Med 143: 634-637, 1973

26. Inoue T, Deporter DA and Melcher AH. Induction of cartilage and bone by dentin demineralized in citric acid. J Perio Res 21: 243-255, 1986

27. Chung JH and Lee JH. Study of bone healing pattern in extraction socket after application of demineralized dentin matrix material. J Korean Assoc Oral Maxillofac Surg 37: 365-374, 2011

28. Tazaki J, Murata M, Yuasa T, Akazawa T, Ito K, Hino J, Nida A, Arisue M and Shibata T. Autograft of human tooth and demineralized dentin matrices for bone augmentation. J Ceram Soc Japan 118: 442-445, 2010

29. Bessho K, Tanaka N, Matsumoto J, Tagawa T and Murata 
Ken Togari et al.: Bone Regeneration by DDM in Skull Defects of Rats

M. Human dentin-matrix-derived bone morphogenetic protein. J Dent Res 70: 171-175, 1991

30. Nebgen DR, Inoue H, Sabsay B, Wei K, Ho CS and Veis A.
Identification of the chondrogenic-inducing activity from bovine dentin (bCIA) as a low-molecular-mass amelogenin polypeptide. J Dent Res 78: 1484-1494, 1999 
J.Hard Tissue Biology Vol. 21(1):25-34, 2012 